\title{
Stretching Food and Being Creative: Caregiver Responses to Child Food Insecurity
}

\author{
Michael P. Burke, PhD, MPH ${ }^{1,2}$; Lauren H. Martini, $\mathrm{MPH}^{2,3}$; Christine E. Blake, PhD, $\mathrm{RD}^{2,4}$; \\ Nicholas A. Younginer, $\mathrm{PhD}^{2,4}$; Carrie L. Draper, $\mathrm{MSW}^{2}$; Bethany A. Bell, MPH, PhD ${ }^{2,5}$; \\ Angela D. Liese, $\mathrm{PhD}^{2,3}$; Sonya J. Jones, $\mathrm{PhD}^{2,4}$
}

\begin{abstract}
Objective: To examine the strategies and behaviors caregivers use to manage the household food supply when their children experience food insecurity as measured by the US Department of Agriculture's Household Food Security Survey Module.

Design: Cross-sectional survey with open-ended questions collected in person.

Setting: Urban and nonurban areas, South Carolina, US.

Participants: Caregivers who reported food insecurity among their children $(\mathrm{n}=746)$.

Phenomenon of Interest: Strategies and behaviors used to manage the household food supply.

Analysis: Emergent and thematic qualitative coding of open-ended responses.

Results: The top 3 strategies and behaviors to change meals were (1) changes in foods purchased or obtained for the household, (2) monetary and shopping strategies, and (3) adaptations in home preparation. The most frequently mentioned foods that were decreased were protein foods (eg, meat, eggs, beans), fruits, and vegetables. The most frequently mentioned foods that were increased were grains and starches (eg, noodles), protein foods (eg, beans, hot dogs), and mixed foods (eg, sandwiches).

Conclusions and Implications: Caregivers use a wide variety of strategies and behaviors to manage the household food supply when their children are food insecure. Future work should examine how these strategies might affect dietary quality and well-being of food-insecure children.
\end{abstract}

Key Words: food insecurity, hunger, children, household food management ( $N$ Nutr Educ Behav. 2017;49:296-303.)

Accepted November 20, 2016. Published online January 7, 2017.

\section{INTRODUCTION}

In 2014 , the children in nearly $11 \%$ of households that had them experienced food insecurity. ${ }^{1}$ Households with food insecurity among children typically report reduced dietary quality, variety, or desirability and may report disrupted eating patterns and reduced food intake. The US Department of Agriculture (USDA) uses the Household Food Security Survey Module (HFSSM) to measure and monitor food insecurity at the household and child levels. ${ }^{1}$ The HFSSM was developed using insights from qualitative interviews with

\footnotetext{
${ }^{1}$ US Department of Agriculture, Food and Nutrition Service, Alexandria, VA

${ }^{2}$ Center for Research in Nutrition and Health Disparities, University of South Carolina, Columbia, SC

${ }^{3}$ Department of Epidemiology and Biostatistics, Arnold School of Public Health, University of South Carolina, Columbia, SC

${ }^{4}$ Department of Health Promotion, Education, and Behavior, Arnold School of Public Health, University of South Carolina, Columbia, SC

${ }^{5}$ College of Social Work, University of South Carolina, Columbia, SC

Conflict of Interest Disclosure: The authors' conflict of interest disclosures can be found online with this article on www.jneb.org.

Address for correspondence: Christine E. Blake, PhD, RD, Department of Health Promotion, Education, and Behavior, Arnold School of Public Health, University of South Carolina, 915 Greene St, Rm 549, Columbia, SC 29208; Phone: (803) 777-1484; Fax: (803) 777-6290; E-mail: ceblake@sc.edu

(C)2016 Society for Nutrition Education and Behavior. Published by Elsevier, Inc. All rights reserved.
}

http://dx.doi.org/10.1016/j.jneb.2016.11.010 adults and caregivers who described what food shortages looked and felt like. ${ }^{2}$ These interviews showed that households used a variety of strategies and behaviors to cope with food insecurity. For example, when food insecurity was at its most severe point, caregivers reported relying on low-cost foods to feed their children or reducing the food intake of their children. Since 1995 when the HFSSM was first implemented nationally, it has been an excellent tool for measuring and monitoring household food insecurity, in large part because of its grounding in research, practice, and widespread adoption.

Although the HFSSM measures broad changes in strategies and behaviors related to managing the dietary quality and quantity of household members, it does not provide information about exact changes to the household food supply. Of particular concern is how food insecurity might affect children's dietary quality and quantity. An assumption embedded in the HFSSM is that caregivers will first sacrifice their 
personal food quality and quantity before they do the same for their children. ${ }^{1,2}$ Little is known, however, about specific changes to children's food quality and quantity when caregivers can no longer buffer the effects of food insecurity. Some work examined the strategies and behaviors caregivers use to obtain more food for the household or make the food that is available last longer when food insecurity occurs (ie, stretching food). For example, relying on federal nutrition programs such as the Supplemental Nutrition Assistance Program (SNAP), food banks, and family and friends is common. ${ }^{3-5}$ No work, however, has specifically examined the behaviors and strategies used when caregivers affirm childreferenced HFSSM items. Examining these strategies and behaviors is important not only for a better understanding of the HFSSM but also for practitioners, researchers, and policy makers who work on nutrition education.

Nutrition education for low-income populations emphasizes strategies and behaviors that maintain adequate dietary quality within a limited food budget. For example, SNAP-Education (SNAP-Ed), the nutrition education component of SNAP has a goal "to improve the likelihood that persons eligible for SNAP will make healthy food choices within

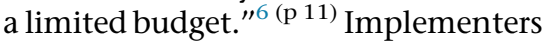
of SNAP-Ed have numerous wellevaluated nutrition education interventions that focus on policy, system, and environmental (PSE) changes to improve nutrition and food security such as increased awareness of federal nutrition assistance programs and social marketing campaigns that encourage healthy eating. ${ }^{7}$ Other resources, typically found through university agricultural extensions, advise families on issues such as using unit pricing to find lowestcost foods, using smaller amounts of meat, poultry, and fish, and planning for leftovers. However, the frequency with which food-insecure caregivers use these strategies and behaviors is not known, especially when dealing with food insecurity among their children.

If nutrition education policies and programming seek to reduce and eliminate food insecurity in children, it is critical first to understand the behaviors and strategies used when caregivers report reductions in dietary quality and quantity among their children. The purpose of this study was to investigate the strategies and behaviors caregivers use to adjust the household food supply in reaction to food insecurity among their children. To serve this purpose, qualitative content analysis was used with a dataset that included responses from caregivers who reported relying on lowcost foods to feed their children or cutting foods from their children's diet because of a lack of financial resources.

\section{METHODS}

This study was part of a larger crosssectional study that investigated causes of low food security in children, formerly known as food insecurity with child hunger. Details of the larger study can be found elsewhere, ${ }^{8}$ but essential components will be summarized here. Data were collected from March, 2012 to May, 2013. This study was approved by the University of South Carolina Institutional Review Board for the Protection of Human Subjects.

\section{Participant Recruitment}

A recruitment-site sampling framework was used that focused on venues where families typically obtain food, with special consideration given to venues that captured households using nutrition assistance programs. These venues were conceptualized in the following manner: (1) traditional venues where families obtained food, such as grocery stores, convenience stores, farmers' markets, dollar stores, daycare centers and other locations that accept SNAP or Special Supplemental Nutrition Program for Women, Infants, and Children benefits; and (2) emergency food assistance venues such as food pantries, food banks, family shelters, and summer feeding sites. One notable exception to the sampling framework was schools, which were excluded because of the administrative burden of working with schools in the study area. Using databases provided by state and local agencies (eg, food banks) and based on previous research in South Carolina,, 10 an initial list of 1,646 potential recruitment sites was generated, which was stratified by urban $(n=776)$ and nonurban $(n=870)$ areas. Next, the researchers used Stata statistical software version 14 (StataCorp LP, College Station, TX; 2014) to select an initial 40 urban and 40 nonurban sites randomly for participant recruitment.
These initial 80 sites were chosen to provide enough variability in types of sites. Finally, the researchers contacted each selected site to ask for permission to recruit. Sites were replaced at random when the site refused to participate, participant recruitment was not successful, or recruitment of new families was exhausted. By the end of the study, 249 urban sites and 178 nonurban sites were contacted; 135 sites yielded screened participants. In addition, some participants were recruited by word of mouth from friends or families that were originally recruited from 1 of the recruitment sites.

At each recruitment site or over the phone, individuals were invited to complete a brief screening questionnaire. Verbal consent was obtained before administering the screener survey. To complete the screener fully and be eligible for the larger study, respondents had to (1) have a child aged $<18$ years living in the household at least $50 \%$ of the time, (2) have a total household income $<\$ 100,000 / y$, and (3) live within an 8-county region in South Carolina. The income limit of $\$ 100,000$ was used because it is about $300 \%$ of the federal poverty threshold for a family of 4 and captures families that are generally ineligible for federal assistance programs but might still struggle financially if they experienced an economic shock severe enough to cause food insecurity. Eligible participants were then administered the USDA 18-item HFSSM.

\section{Measures}

Demographic information. As part of the screener, limited demographic information was collected. In addition to the eligibility criteria already mentioned, each participant reported his or her race and ethnicity and home address. Based on this information, a race and ethnicity variable (non-Hispanic white, non-Hispanic African American, or other) was constructed. The other race category was not further defined because few participants selected this category (2.3\%). Using a respondent's home address, an urbanicity variable was constructed. Participants who lived in a city center were coded as urban and those who lived outside a city center were coded as nonurban.

Household food security status. The USDA's 18-item HFSSM was used to assess household food security status. 
The HFSSM measures experiences with worrying about food shortages, reduced quality and quantity of food in the household, and hunger among both adults and children in the previous 12 months. The food security status of households was classified in the following manner: (1) food secure (affirming $\leq 2$ items), (2) food insecure (affirming $\geq 3$ items but $<5$ of the child-level items; this corresponded to the USDA label of low food security without very low food security in children), or (3) very low food security (VLFS) among children (affirming $\geq 5$ childlevel items). The HFSSM is a standard measure of food security in the US and is valid, reliable, and widely used. ${ }^{1,2,11}$

\section{Follow-up Questions}

Two follow-up questions were added to the end of the HFSSM if participants affirmed certain child-level HFSSM items. The purpose of these follow-up questions was to elicit the strategies and behaviors caregivers used to adjust the household food supply in reaction to food insecurity among their children. These 2 follow-up questions were pilottested in a small sample of caregivers $(\mathrm{n}=10)$. Pilot-testing included reading the question to the participant, who was allowed to comment on the clarity, comprehension, and relevance of the question. The results of the pilot-testing were used to assess whether the questions needed to be modified. Results are described subsequently along with a description of each follow-up question.

Reliance on low-cost foods to feed children. Participants who affirmed the HFSSM item: We relied on only a few kinds of low-cost food to feed our children because we were running out of money to buy food, were asked an open-ended follow-up question: You mentioned that sometimes your family has to rely on a few low-cost foods to feed your children because there was not enough money to buy food. How do you change the meals in your household when this occurs? A variant of this question was pilot-tested, in which instead of asking: How do you change the meals in your household when this occurs? participants were asked, Tell me about the low-cost foods you rely upon. Instead of describing specific low-cost foods, participants would describe how they changed their children's meals and did not focus solely on low-cost foods. Therefore, the research team decided that the follow-up question should focus on changes to meals rather than specific low-cost foods.

Cutting children's meal size. Participants who affirmed the item: In the past 12 months, did you ever cut the size of any of the children's meals because there was not enough money for food? were asked an open-ended follow-up question: You mentioned that sometimes you have to cut the size of your children's meals because there was not enough money for food. Tell me about the foods you usually cut. This question performed well in pilot-testing and was not modified.

\section{Analytic Approach}

Participant responses to each follow-up question were manually typed in real time into a database during the screening process. Four master's-level or doctoral students in public health or social work collected participant responses. The students received in-person and in-field training in qualitative data collection from a Master of Social Work researcher with previous qualitative date collection experience. Furthermore, except for 1 person, study staff had completed at least 1 research methods course as a part of their field of study, which included qualitative methods. The 1 person, who helped complete screening surveys without this level of education, received extensive training in the field before conducting data collection solo. Typed responses were exported to Microsoft Excel 2016 (Microsoft Corporation, Redmond, WA). Participant responses to each follow-up question were used as the source for participant strategies and behaviors. Emergent coding ${ }^{12}$ was used to identify strategies and behaviors caregivers used to adjust the household food supply in reaction to child food insecurity. Three researchers coded the qualitative data. All steps in the coding process involved multiple coders and extensive peer review of themes and assignment of text passages. One coder (LHM) conducted the initial emergent coding to identify strategies and behaviors caregivers used to adjust the household food supply in reaction to child food insecurity. A second coder (CEB) reviewed the coding and adjusted the emergent code list. A third coder (MPB) reviewed the emergent code list and provided guidance regarding the interpretation of passages and code list. During the emergent coding process, it became clear that participants mentioned strategies and behaviors for cutting children's meal size when asked about relying on low-cost foods, and vice versa. This was true even when participants answered only 1 of the follow-up questions. Many participants described the same strategies or behaviors in reaction to relying on low-cost foods or cutting the size of their children's meals. Therefore, responses to the 2 questions were merged and coded for strategies and behaviors across all responses. Participants could list multiple strategies or behaviors per follow-up question. Upon completion of emergent coding, the code list and the full coded data set was shared with the entire research team. The research team then reviewed the code list and assignment of passages to codes and provided further revisions and guidance on next steps.

The first coder then used thematic coding techniques ${ }^{12}$ to place strategies and behaviors into 2 categories: (1) broad strategies and behaviors that affected the overall household food supply; and (2) behaviors related to decreasing or increasing specific foods. Within each of these categories, primary and subcategories were coded. For example, the primary category changes in foods purchased or obtained for household meals was created, which was further refined into the subcategories prioritize some foods (eg, hot dogs, noodles, chicken, rice, beans, potatoes, bread and grits) and use foods that can be stretched (eg, stews, soups, casseroles, pasta, bean or rice dishes). As another example, the primary category increasing or decreasing dairy was created, which was further refined into the subcategories milk, cheese, and yogurt. The second coder (CEB) again reviewed the coding and adjusted codes after discussion with the full coding team (LHM and MPB). The number of instances when respondents mentioned the primary and subcategories was counted, as well as their relative frequencies (ie, number of instances a strategy or behavior was mentioned divided by the total number of strategies 
and behaviors mentioned). At this point the new code list, the full coded data set, and preliminary findings were again shared with the entire research team. The purpose of this analysis methodology was to enumerate the qualitative strategies and behaviors respondents used while maintaining the integrity of the respondents' own experiences.

\section{RESULTS}

A total of 746 participants affirmed at least 1 of the 2 child food insecurity items; 11 provided responses to the follow-up question about cutting foods only; 221 provided responses to the follow-up question about changes to meals only; and 514 provided responses to both follow-up questions (Table 1). Thirty-five percent of the sample had VLFS among children; $61 \%$ lived in an urban area; and $80 \%$ were African American. The authors initially stratified the analysis by food security status (food-insecure households in which children were experiencing VLFS compared with food-insecure households in which children were not experiencing VLFS).
Upon comparing the stratified with nonstratified analyses, the authors found the frequency of strategies and behaviors to be nearly identical with no considerable differences; therefore, only the nonstratified analysis are presented.

Participants mentioned a total of 1,354 strategies and behaviors to adjust the household food supply in reaction to child food insecurity; these were grouped into 5 primary categories $(\mathrm{n}=735)$ (Table 2). Changes in foods purchased or obtained for household's meals was the most frequent primary strategy or behavior mentioned $(n=773)$ and had the greatest share of total mentions (57\%). Common subcategories within this category included prioritize some foods (eg, hot dogs, noodles, chicken, rice, beans, potatoes, bread and grits $)(\mathrm{n}=239 ; 17 \%)$ and use foods that can be stretched (eg, stews, soups, casseroles, pasta, bean or rice dishes) $(\mathrm{n}=206 ; 15 \%)$. Monetary and shopping strategies was the second most frequent primary strategy or behavior mentioned $(\mathrm{n}=245)$. Common subcategories within this category included buy according to price (eg, sale, cheapest and store brands) $(\mathrm{n}=191 ; 14 \%)$ and shop at

Table 1. Sociodemographic Characteristics of Caregivers Who Affirmed Specific Child-Level Items in US Department of Agriculture's Household Food Security Module (HFSSM) $(n=746)$

\begin{tabular}{lr} 
Sociodemographic Characteristic & Caregivers (n [\%]) \\
Food security & \\
$\quad$ Food insecure & $480(64.3)$ \\
$\quad$ Very low food security among children & $266(35.7)$ \\
Urbanicity & \\
$\quad$ Non-urban & $290(38.8)$ \\
$\quad$ Urban & $456(61.1)$ \\
Race/ethnicity & \\
$\quad$ Non-Hispanic African American & $601(80.6)$ \\
$\quad$ Non-Hispanic white & $128(17.2)$ \\
$\quad$ Other & $17(2.3)$ \\
Household food security child-level items affirmed & \\
$\quad$ Reliance on low-cost food item only & \\
$\quad$ Cutting children's meal size item only & \\
$\quad$ Both reliance on low-cost food and cutting children's & $11(1.5)$ \\
$\quad$ meal size items & $514(68.9)$ \\
\hline
\end{tabular}

${ }^{a}$ Food-insecure households affirmed $\geq 3$ items in the 18-item HFSSM but did not have very low food security among children. Very low food security among children households affirmed $\geq 5$ child-reference items in the HFSSM; ${ }^{b}$ Full item text: We relied on only a few kinds of low-cost food to feed our children because we were running out of money to buy food. Was that often, sometimes, or never true for you in the past 12 months?; 'Full item text: In the past 12 months, did you ever cut the size of any of the children's meals because there was not enough money for food? (Response options: yes or no.)

budget stores ( $\mathrm{n}=30 ; 2 \%)$. Changes in household meal patterns was the third most frequent primary strategy or behavior mentioned $(\mathrm{n}=194$; 14\%). Common subcategories within this category included serve smaller portions at meals $(\mathrm{n}=125 ; 9 \%)$ and cut adult portions or adult does not eat at all $(n=22 ; 2 \%)$. Adaptations in home preparations was the fourth most frequent primary strategy or behavior ( $n=130 ; 9 \%$ ). Common subcategories within this category included be creative/make stuff up/use what we have $(\mathrm{n}=92 ; 6 \%)$ and make use of leftovers and freeze meals $(\mathrm{n}=21 ; 2 \%)$.

Caregivers reported a total of 1,365 behaviors related to decreasing or increasing specific foods in their children's meals; these were grouped into 11 primary categories $(\mathrm{n}=525)$ (Table 3). Protein foods were the most commonly mentioned decreased food $(\mathrm{n}=322 ; 57 \%)$ : specifically meat (nonspecific type) $(\mathrm{n}=254 ; 45 \%)$, beef ( $\mathrm{n}=14 ; 2.5 \%)$, chicken or turkey $(\mathrm{n}=22 ; 3.9 \%)$, and pork or bacon $(\mathrm{n}=10 ; 1.8 \%)$. Vegetables were the second most commonly mentioned decreased food $(n=66 ; 11 \%)$, followed by grains and starches $(\mathrm{n}=63 ; 11 \%)$. Within grains and starches, commonly mentioned decreased foods were breads $(n=17$; $3 \%)$ and rice $(\mathrm{n}=20 ; 3.5 \%)$. The least likely primary behaviors for decreasing foods were decreasing baby food or formula ( $\mathrm{n}=3 ;<1 \%)$ and decreasing fats or oils $(\mathrm{n}=2 ;<1 \%)$. Grains and starches were the most commonly mentioned increased foods $(\mathrm{n}=365 ; 45 \%)$, specifically noodles ( $\mathrm{n}=140 ; 17 \%)$ and rice ( $n=76 ; 9 \%$ ). Protein foods were the second most commonly mentioned increased foods ( $\mathrm{n}=208 ; 26 \%)$, specifically hot dogs $(n=47 ; 6 \%)$ and beans $(n=52$; $6 \%$ ). Mixed dishes were the third most commonly mentioned increased foods $(\mathrm{n}=152 ; 19 \%)$, specifically sandwiches (unknown type) $(\mathrm{n}=40$; $5 \%)$, cold cut sandwiches $(n=28 ; 3 \%)$, and peanut butter and jelly sandwiches $(n=40 ; 5 \%)$. The least likely foods to be increased were fruits $(\mathrm{n}=4 ;<1 \%)$ and dairy $(\mathrm{n}=12 ; 1 \%)$.

\section{DISCUSSION}

This study found that caregivers who reported food insecurity among their children used many strategies and behaviors previously documented in the 
Table 2. Strategies and Behaviors Caregivers Used to Adjust Household Food Supply in Reaction to Food Insecurity Among Their Children $(n=735)$

\section{Strategies and Behaviors Used to Change Children's Meals}

Changes in foods purchased or obtained for household meals

Prioritize some foods (eg, hot dogs, noodles, chicken, rice, beans, potatoes, bread and grits)

Use foods that can be stretched (eg, stews, soups, casseroles, pasta, bean or rice dishes)

Eat more sandwiches to simplify meals

Use more canned goods

Cut quantity and quality of meats

Food banks, church food pantries, food giveaways

Borrow food or money (eg, from family, friends, neighbors)

Cut some foods (exact foods not specified)

More processed and fast foods over fresh foods (eg, boxed and canned dinners, dollar menus)

Child food preferences come first

Use of federal nutrition assistance programs

Garden to supplement food supply

Monetary and shopping strategies

Buy according to price (eg, sale, cheapest and store brands)

Shop at budget grocery stores (e.g., Walmart, Aldi, Save-a-lot)

Buy foods in bulk, especially meats

Budget finances to stretch funds over a month

Use coupons

Use utility and other bill money for food purchases

Sell plasma for money to buy food

\section{Times Strategy} Mentioned

773

239

206

64

56

54

45

40

28

23

10

5

3

245

191

30

14

4

3

2

1

194

125

22

21

14

7

4

1

130

92

21

7

5

5

12
Times Mentioned (\%) 57.1 17.7

15.2

4.6

4.1

4.0

3.3

2.9

2.0

1.7

0.7

0.4

0.2

18.1

14.1

2.2

1.0

0.3

0.2

0.1

0.1

14.3

9.2

1.6

1.5

1.0

0.5

0.3

0.1

9.6

6.6

1.6

0.5

0.4

0.4

0.8

Try to make do/do not know/do not cut back

Note: Each participant could list multiple strategies and behaviors. There were 1,354 total strategies and behaviors mentioned from 735 participants. Primary strategies and behaviors, as well as subcategories, are listed in descending order. Caregivers were asked, You mentioned that sometimes your family has to rely on a few low-cost foods to feed your children because there was not enough money to buy food. How do you change the meals in your household when this occurs? or, You mentioned that sometimes you have to cut the size of your children's meals because there was not enough money for food. Tell me about the foods you usually cut, if they affirmed either: We relied on only a few kinds of low-cost food to feed our children because we were running out of money to buy food. Was that often, sometimes, or never true for you in the past 12 months? or, In the past 12 months, did you ever cut the size of any of the children's meals because there was not enough money for food? (response options: yes or no) in the US Department of Agriculture's Household Food Security Survey Module.

literature. For example, Kempson and colleagues $^{3}$ used data collected from focus groups with low-income individuals to categorize strategies and behaviors in the following manner: relying on community resources, interacting with informal support systems, supplementing financial resources, lowering food costs by using shopping strategies, managing the household food supply, and regulating eating patterns. Anater and colleagues ${ }^{4}$ collected survey data from a sample of low-income, mostly food-insecure individuals who were using emergency food assistance. They 
Table 3. Foods Decreased or Increased in Children's Meals by Caregivers in Reaction to Food Insecurity Among Their Children $(n=525)$

\section{Food Groups}

Total mentions of decreasing or increasing foods (\%)

All foods

Baby food or formula

Dairy

Milk

Cheese

Yogurt, pudding, or ice cream

All dairy products

Fats or oils

Fruits

Fruit

Fresh fruit

Fruit juice

Grains and starches

Breads

Rice

Cereals

Potatoes

Oatmeal

Pasta

Noodles

Pancakes

Grits

Crackers or popcorn

All grains and starches

Less healthy food and sweets

Snacks

Less healthy food

Dessert or sugar

Sugar-sweetened beverages

French fries or potato chips

Mixed foods

Cold cut sandwiches

Peanut butter and jelly sandwiches

Any sandwich

Burritos, tacos, pizza, Hot Pockets, or TV dinners

Casseroles, canned entrees, canned foods, or hash

Soup or chili

Hamburger Helper or Tuna Helper or macaroni and cheese

Side items

Protein foods

Meat (nonspecific type)

Eggs

Beans

Fish

Hot dogs

Peanut butter

Beef

Chicken or turkey

Pork or bacon

Vegetables

Vegetables
Decrease

Increase

$\begin{array}{cc}563 & 802 \\ 5(0.9) & 0\end{array}$

$3(0.5)$

$21(3.7)$

$14(2.5)$

$2(0.4)$

$3(0.5)$

$2(0.4)$

$2(0.4)$

$32(5.7)$

$20(3.5)$

$4(0.7)$

8 (1.4)

63 (11.2)

17 (3.0)

$20(3.5)$

$6(1.1)$

7 (1.2)

0

$2(0.4)$

$1(0.2)$

0

0

0

$10(1.8)$

$34(6.0)$

$10(1.8)$

$6(1.1)$

9 (1.6)

7 (1.2)

$2(0.4)$

$16(2.8)$

$2(0.4)$

0

$1(0.2)$

$4(0.7)$

$1(0.2)$

0

$3(0.5)$

$5(0.9)$

$321(57.1)$

254 (45.0)

$6(1.1)$

$4(0.7)$

5 (0.9)

$4(0.7)$

$2(0.4)$

$14(2.5)$

22 (3.9)

$10(1.8)$

$66(11.7)$

57 (10.1)
0

0

$12(1.4)$

$8(0.9)$

$3(0.3)$

0

$1(0.1)$

0

$4(0.4)$

$4(0.4)$

0

0

365 (45.5)

$33(4.1)$

$76(9.4)$

27 (3.3)

27 (3.3)

$8(0.9)$

$17(2.1)$

140 (17.4)

$2(0.2)$

29 (3.6)

$4(0.4)$

$2(0.2)$

$14(1.7)$

$2(0.2)$

0

$3(0.3)$

$6(0.7)$

$3(0.3)$

152 (18.9)

28 (3.4)

22 (2.7)

$40(4.9)$

16 (1.9)

$10(1.2)$

17 (2.1)

19 (2.3)

0

208 (25.9)

$8(0.9)$

$12(1.4)$

$52(6.4)$

$12(1.4)$

47 (5.8)

$3(0.3)$

21 (2.6)

$43(5.3)$

$10(1.2)$

$40(4.9)$

30 (3.7)

(continued) found that the top 3 strategies and behaviors engaged in during the 12 months before survey administration were going to a food pantry, buying foods because they were on sale, and making meals with low-cost foods. Other work among low-income and food-insecure populations showed common strategies and behaviors that include relying on food banks; using coupons or buying discounted foods; shopping at low-cost stores; relying on friends, family, and neighbors; stretching existing food over more meals; eating less; budgeting household financial resources; and using federal nutrition assistance programs. ${ }^{13,14}$ A distinction between the current study and previous ones is that only caregivers whose children were experiencing food insecurity were included, and strategies and behaviors were only asked about when caregivers affirmed certain HFSSM items. Previous studies instead asked about strategies and behaviors among participants with and without children, among those who were food secure or food insecure, and not in response to HFSSM items. Given these differences, it was possible for the current study to find strategies and behaviors distinct from those reported previously. The strategies and behaviors used by caregivers of food-insecure children, however, were similar to those in other studies with more demographically inclusive populations. It is possible that the strategies and behaviors individuals use in reaction to food shortages remain the same regardless of who in the household is affected. Future work should examine strategies and behaviors used in reaction to food insecurity within households with and without children, as well as in households with and without food insecurity among children.

Caregivers reported a variety of changes to their children's meals in reaction to food insecurity. For example, the most commonly decreased foods in reaction to food insecurity among children were protein foods, specifically meat, followed by fruits and vegetables. The most commonly increased foods to cope with food insecurity among children were grains and starches (mostly noodles) and low-cost protein foods, particularly beans and hot dogs. The types of foods increased or decreased in reaction to food insecurity in children have several implications. First, the literature on dietary quality of 
Table 3. Continued

\section{Food Groups}

Canned vegetables

Fresh vegetables

Water

Notes: Each participant could list multiple increases or decreases within and across each of the food groups. There were 1,365 total decreases or increases mentioned from 525 participants. Primary food group categories are listed alphabetically; subcategories are listed in descending order. Caregivers were asked, You mentioned that sometimes your family has to rely on a few low-cost foods to feed your children because there was not enough money to buy food. How do you change the meals in your household when this occurs? or, You mentioned that sometimes you have to cut the size of your children's meals because there was not enough money for food. Tell me about the foods you usually cut, if they affirmed either: We relied on only a few kinds of low-cost food to feed our children because we were running out of money to buy food. Was that often, sometimes, or never true for you in the past 12 months? or, In the past 12 months, did you ever cut the size of any of the children's meals because there was not enough money for food? (response options: yes or no) in the US Department of Agriculture's Household Food Security Survey Module.

children living in food-insecure households is mixed, and the current study might help guide future work in this area. Hanson and Connor ${ }^{15}$ did a systematic review of the literature on the association between children's dietary quality and household food insecurity. They found substantial evidence for lower fruit consumption among children in food-insecure households but less evidence for grains, vegetables, dairy, and sweets. Furthermore, according to Hanson and Connor, only $16 \%$ of studies found an adverse association between dietary quality and food insecurity. Caregivers reported decreasing and increasing a wide range of foods in their children's diets to cope with food insecurity, and the effects these changes have on children's diets likely vary substantially. For example, decreasing some protein foods and increasing other protein foods were commonly reported reactions to food insecurity among children. Many caregivers also reported decreasing fruits and vegetables in reaction to food insecurity in their children. Children in low-income households do not meet fruit and vegetable intake, ${ }^{16}$ and the current results suggested that child food insecurity might contribute to this disparity. The effects of the different kinds of changes on overall diet quality, physical health, and caregiver and child psychological wellbeing are not known. It is possible that families that decrease consumption of red meats with high saturated fats and replace them with a healthier protein (eg, legumes) have a net gain in overall nutritional quality. Nevertheless, the possible negative psychological effect of cutting meat or using low-cost proteins within the context of household food insecurity also must be considered. Many adults view meat as a necessary part of each meal, and its involuntary absence is considered detrimental to well-being. ${ }^{17,18}$ Changes to dietary quality because of food insecurity may have either positive may also have psychological implications. Future work should consider the exact changes to household members' diets in reaction to food insecurity and how they affect not only dietary quality but also psychological well-being.

This study had limitations. First, the number of times a participant mentioned a strategy or behavior was measured, but not the extent to which each behavior occurred. For example, participants might have mentioned that they used coupons or increased breads, but it is not known how often they used coupons or how much they increased bread consumption. In addition, it is not known how long households used these strategies and behaviors. It is possible that households adapt their strategies and behaviors over time. Future work should quantify these behaviors and strategies to capture their frequency and extent better, as well as examine whether they change over time. Second, the researchers used a or negative effects on dietary quality but qualitative approach in this study. Qualitative methods are appropriate when the research questions seek to understand participants' experiences or perceptions and to capture these perceptions in their own words. The strategies that emerged from data collected in this study were from open-ended questions that were not designed to provide insight into statistical frequencies or differences. Future work could build upon the current findings using quantitative methods and examine whether there are significant difference in strategies and behavior among population groups and how these differences might relate to nutritional outcomes. Third, the sampling framework used was not meant to be representative of any population, but rather to be large and diverse enough to capture considerable variation in participant characteristics. Therefore, findings from this might not be generalizable to other food-insecure households or populations. Finally, data were collected in person and on location during recruitment. In other words, it was not possible to do formal in-depth interviews with respondents to gain a deeper understanding of strategies and behaviors. In addition, some respondents might have not felt comfortable answering questions in front of the interviewer, especially because of the social stigma of not being able to afford enough food for children in the household.

This study also had strengths. First, strategies and behaviors were examined among food-insecure households with children, all of whom had some indication of child food insecurity as indicated by specific HFSSM items. To the best of the authors' knowledge, no other study has examined this population in such a way, and it is their opinion that this study provides valuable insights into the strategies and behaviors used in reaction to child food insecurity. Second, participants could use their own words and thoughts when answering the questions. This allowed a wide range of strategies and behaviors to be captured from the perspective of those that perform them. Third, each participant was asked the same questions, allowing for standardization in data collection. Finally, this study provides insight into how participants interpret certain child HFSSM items, which is relevant for refinement of the HFSSM. 


\section{IMPLICATIONS FOR PRACTICE AND RESEARCH}

Common strategies and behaviors suggested by nutrition educators to consume a healthy diet on a limited budget include making ingredient substitutions, using protein alternatives, and shopping for sales or coupons. Caregivers of food-insecure children were already found to be using many of these suggested strategies and behaviors. This study showed that such nutrition education suggestions reflect practiced strategies and behaviors within a food-insecure population. However, these findings could also show that these strategies and behaviors are not sufficient to buffer against the effects of having a limited budget in relation to food insecurity. That is, although many respondents used these strategies and behaviors, they were still food insecure (although possibly less food insecure than they would have been if using none of the strategies or behaviors). Although person-level SNAP-Ed is effective in reducing food insecurity, ${ }^{19}$ it is likely that a more comprehensive approach to addressing food insecurity in children through nutrition education is needed. The PSE changes have become a recommended strategy for use in public health and SNAP-Ed, and many nutrition education interventions that use PSE strategies would likely increase food security as well. For example, the SNAP-Ed toolkit, ${ }^{7}$ which provides practitioners with numerous PSE strategies, suggests increasing access to school federal nutrition assistance programs, supporting development of wellness councils, promoting community gardens, and increasing access to healthy food in retail settings. Each of these strategies may strengthen and support individual strategies and behaviors in reaction to food insecurity. Future research could examine whether PSE strategies strengthen individual strategies and behaviors in reaction to food insecurity.

\section{ACKNOWLEDGMENTS}

This project was supported with a grant from the University of Kentucky Center for Poverty Research through funding by the US Department of Agriculture, Food and Nutrition Service, Contract No. AG-3198-B-10-0028. In addition, this study received funding from the $\mathrm{Na}$ tional Institute of Health - National Institute of General Medical Science (T32GM081740). Michael P. Burke completed this study as a doctoral student at the Department of Health Promotion, Education, and Behavior, Arnold School of Public Health, University of South Carolina. The opinions and conclusions expressed herein are solely those of the authors and should not be construed as representing the opinions or policies of the University of Kentucky Center for Poverty Research, the US Department of Agriculture, Food and Nutrition Service, or the National Institute of Health - National Institute of General Medical Science.

\section{REFERENCES}

1. Coleman-Jensen A, Rabbitt MP, Gregory C, Singh A. Household Food Security in the United States in 2014. Washington, DC: US Dept of Agriculture, Economic Research Service; 2015.

2. National Research Council. Food Insecurity and Hunger in the United States: An Assessment of the Measure. Washington, DC: National Academies Press; 2006.

3. Kempson K, Keenan DP, Sadani PS, Adler A. Maintaining food sufficiency: coping strategies identified by limitedresource individuals versus nutrition educators. J Nutr Educ Behav. 2003;35: 179-188.

4. Anater A, McWilliams R, Latkin C. Food acquisition practices used by food-insecure individuals when they are concerned about having sufficient food for themselves and their households. $J$ Hunger Environ Nutr. 2011;6:27-44.

5. Eikenberry N, Smith C. Attitudes, beliefs, and prevalence of dumpster diving as a means to obtain food by Midwestern, low-income, urban dwellers. Agric Human Values. 2005;22:187-202.

6. United States Department of Agriculture. Supplemental Nutrition Assistance Program Education Plan Guidance FY 2017-Nutrition Education \& Obesity Prevention Grant Program. Washington, DC: US Dept of Agriculture; 2016.

7. US Department of Agriculture. SNAPEd Strategies \& Interventions: An Obesity Prevention Toolkit for States. Alexandria,
VA: US Dept of Agriculture, Food and Nutrition Service; 2016.

8. Liese AD, Draper CL, Martini L, et al. Recruitment strategies and participation in a study of childhood hunger [published online ahead of print May 5, 2016]. J Hunger Environ Nutr. http://dx. doi.org/10.1080/19320248.2015.1112760.

9. Liese AD, Colabianchi N, Lamichhane AP, et al. Validation of 3 food outlet databases: completeness and geospatial accuracy in rural and urban food environments. Am J Epidemiol. 2010;172:1324-1333.

10. Liese AD, Barnes TL, Lamichhane AP, Hibbert JD, Colabianchi N, Lawson AB. Characterizing the food retail environment: impact of count, type, and geospatial error in 2 secondary data sources. $J$ Nutr Educ Behav. 2013;45:435-442.

11. Gundersen C, Ziliak JP. Food insecurity and health outcomes. Health Aff. 2015; 34:1830-1839.

12. Hsieh H, Shannon SE. Three approaches to qualitative content analysis. Qual Health Res. 2005;15:1277-1288.

13. Ahluwalia IB, Dodds JM, Baligh M. Social support and coping behaviors of low-income families experiencing food insufficiency in North Carolina. Health Educ Behav. 1998;25:599-612.

14. Jarrett OL, Bahar OS, OdomsYoung A, Jarrett RL, Sensoy Bahar O, Odoms-Young A. "You just have to build a bridge and get over it": Lowincome African American caregivers' coping strategies to manage inadequate food supplies. J Poverty. 2014;18: 188-219.

15. Hanson KL, Connor LM. Food insecurity and dietary quality in US adults and children: a systematic review. Am J Clin Nutr. 2014;100:684-692.

16. Lorson BA, Melgar-Quinonez HR, Taylor CA. Correlates of fruit and vegetable intakes in US children. $J$ Am Diet Assoc. 2009;109:474-478.

17. Szurek SM. Social identity and food choice in the Southeastern Unite States. Nutr Anthr. 2005;27:23-37.

18. Douglas M. Deciphering a Meal. In: Counihan C, Van Esterik P, eds. Food and Culture: A Reader. New York, NY: Routledge; 1997:36-54.

19. Eicher-Miller HA, Mason AC, Abbott AR, McCabe GP, Boushey CJ. The effect of food stamp nutrition education on the food insecurity of lowincome women participants. J Nutr Educ Behav. 2009;41:161-168. 


\section{CONFLICT OF INTEREST}

The authors have not stated any conflicts of interest. 